\title{
RESEÑA: THE ROAD FROM GEORGE ORWELL: HIS ACHIEVEMENT AND LEGACY. ALBERTO LÁZARO, ED.
}

\author{
Alejandro Melero Salvador. Universidad de Almería
}

Lázaro, Alberto, ed. 2001. The Road from George Orwell: His Achievement and Legacy. Bern: Lang. 250 páginas. ISBN 3-906766-79-9.

Nada demuestra tanto la vigencia de un autor como el hecho de que, pasadas décadas desde su muerte, siga generando polémica y discusión. Que George Orwell es una de las figuras claves de la literatura inglesa del siglo XX lo prueban los numerosos estudios que se le dedican cada año; que su pensamiento sigue vivo pudo verse en las VIII Conferencias sobre Literatura Inglesa de la Universidad de Alcalá (2000), de donde nace este libro en el que, junto a tradicionales enfoques en la obra de Orwell, se esbozan nuevas perspectivas críticas que no hacen sino demostrar la actualidad de la literatura de este excepcional autor, sobre el que las aún nacientes orientaciones postmodernistas o feministas tienen mucho que decir. El libro está dividido en dos claras secciones de seis artículos cada una, dedicándose la primera a revisar su obra y la segunda a rastrear la influencia de ésta en autores contemporáneos. En todo el conjunto se aprecia además un especial interés por rescatar el olvidado valor formal del genial autor de Nineteen Eighty-Four.

El primero de los artículos, "The Road to Utopia, or on Orwell's Idealism" de Fernando Galván, es también uno de los más interesantes. En él se analizan las contradicciones orwellianas como clave para entender su obra. Sus contradicciones como autor y como crítico, y también como persona, se deben según Galván a su espíritu idealista y revolucionario, y a su miedo a quedar atrapado en ideas pre-establecidas. Es Orwell un hombre de acción y no de razón. Y es en sus años y experiencia en España donde más claramente podemos entender este carácter rebelde y contradictorio. La Guerra Civil en la que Orwell tanto ansió luchar, a pesar de los consejos que Henry Miller le diera, supuso un punto de inflexión en su actitud idealista. En España conoció la susceptibilidad de cualquier ideología a ser corrupta y el desengaño del idealista. Tal vez por este desengaño es por lo que Galván establece una acertadísima y muy documentada comparación entre Orwell y Don Quijote. El espíritu que incita al caballero andante a iniciar su viaje también invadió a Orwell en sus escapadas a París, Londres y España, así como a seguir luchando una vez acabada la guerra, como haría Don Quijote enfermo en cama. La humanidad quijotesca en su labor de desfacer entuertos es también orwelliana, como demuestra su conocida anécdota 
en las trincheras, cuando fue incapaz de disparar al enemigo fascista por considerarle "a fellow-creature, similar to yourself".

El siguiente artículo, “A Revolutionary in Love with the 1900s: Orwell in Defence of "Old England" continúa analizando las contradicciones en el pensamiento orwelliano a partir de su novela Coming up for Air (1939) y de algunos de los ensayos compilados y estudiados por Sonia Orwell (Essays, Journalism and Letters ), manejados con seguridad por la autora de este artículo. Chirstine Berberich se pregunta cómo es posible que un revolucionario como Orwell sintiese una devoción tan romántica por los valores tradicionales ingleses. Efectivamente, la idea de ser socialista está reñida con la de ser un patriota convencional, enamorado de la Inglaterra de principios de siglo. Berberich se esfuerza por dilucidar la distinción entre patriotismo y nacionalismo y con ello nos demuestra la vigencia de las ideas de Orwell, que siempre fue consciente del peligro del nacionalismo sin por ello renunciar a un profundo apego por su país. Así pues, frente al sobrecogimiento nacionalista, Orwell propone un patriotismo activo que ensalce los valores positivos de Inglaterra en detrimento de los negativos. Sus constantes viajes por Inglaterra y sus estancias en el extranjero legitiman su valor como crítico con su país pues le dan la distancia suficiente para ser, si no objetivo, conocedor de otras perspectivas. Berberich rastrea en la obra de Orwell la exaltación de valores tradicionales ingleses (comida, té, "English gentleness") pero no descuida la censura del autor a los aspectos menos afortunados de su tierra natal (incluyendo su triste clima o su inexistente tacto para atraer al turista).

Otra de las contradicciones exploradas por Berberich es la admiración de Orwell por los "good old days" mientras regaña a sus coetáneos por vivir en el pasado. Una vez más su impulso revolucionario lucha contra unos valores demasiados arraigados para ser arrancados con facilidad. La solución a esta paradoja puede estar quizá en el acertado juicio de Fernando Galván en el artículo anterior, cuando explica que Orwell es, ante todo, un hombre de acción. No podemos evitar recordar que Pío Baroja, el otro "hombre de acción" y contemporáneo de Orwell, también sufrió el dilema de un profundo amor por una "patria" en la que no dejaba de ver defectos. Berberich, sin embargo, justifica este afecto irreprimible hacia los días pasados explicando que la infancia y educación del Orwell niño transcurrieron próximas a las clases más acomodadas, donde le enseñaron a amar y respetar unos valores por los que el autor nunca dejaría de sentir nostalgia. Sea como fuere, todas estas distintas posturas en la vida ideológica de Orwell enseñan al lector de comienzos del siglo XXI que "Orwell was nostalgic about the past, yes. But he also, more than anything, wanted social changes. He wanted the people of England to wake up and take political responsibility (...) But he did not want to do so at all cost; he wanted to keep certain forms, certain values that had proved valuable in the past and wanted to apply them to the present" (51).

Los capítulos de Miquel Berga y Alberto Lázaro estudian la relación de Orwell con nuestra Guerra Civil. Berga, en “Orwell's Catalonia Revisited: Textual Strategies and the Eyewitness Account", analiza su Homage to Catalonia (1938) como una obra con tanto valor histórico como literario. Parece estar muy interesado en la valía de este texto para el historiador. Indaga en la escritura consciente de un Orwell que sabía que la "story" que estaba escribiendo era también "history". Berga se preocupa por la fiabilidad de un novelista trabajando como reportero, y no descuida "the fictional potential of what is presented as autobiographical reportage" (58). Este artículo nos ofrece el interesante documento gráfico del propio Orwell en las filas republicanas, unas fotografías que Berga pretende utilizar como testimonio visual y prueba de la verosimilitud del autor como un testigo fidedigno. Para Berga, el hecho de que Homage to Catalonia posea tan grandes

Odisea, $n^{\circ} 2,2002$ 
dosis de valor literario e histórico radica en la tremenda habilidad de Orwell para ser un ejemplar escritor-artesano y de su impresionante dominio de la forma literaria. Esquematiza esta maestría literaria en siete "estrategias textuales" (uso del detalle realista, la interrupción del discurso narrativo por el propio autor dirigiéndose al lector...) a las que se les podrían haber sumado otras tantas, como, por ejemplo, la humanización de todos los personajes, incluyendo al enemigo, o el intento de objetividad al evitar la inevitable distinción "buenosmalos".

Alberto Lázaro ("George Orwell's Homage to Catalonia: A Politically Incorrect Story") trata con extrema destreza la significativa cuestión de la dificultad que tuvo Orwell para ver su Homage to Catalonia publicada. Todo este artículo de investigación respira la solidez que le otorga su precisa documentación. En primer lugar, ofrece una interesante indagación en el sistema censor franquista que nos introduce muy bien en los problemas que esta obra debió de tener en nuestro país. Descubrimos aquí que la crítica al socialismo corrupto, tan presente en toda su obra, fue el mejor de los pasaportes para que Homage to Catalonia pudiese publicarse en la España de los 60, incluso en catalán (Homenatge a Catalunya: un testimoni sobre la Revolució Espanyola, por la editorial Ariel). La precisión y el detalle con los que Lázaro contrasta el original con la versión divulgada tras pasar la censura hacen que su esquema comparativo entre una y otra sea imprescindible para entender la recepción de este libro en nuestro país. Con gran acierto, Lázaro no termina ahí sino que continúa escudriñando los problemas que este texto tuvo en la propia Inglaterra, donde (en este caso por parte de sectores de izquierdas) también tuvo una ardua y tardía publicación. Termina el artículo criticando la situación actual, pues, asegura, los errores del pasado no se han corregido y todavía es difícil encontrar una traducción correcta al español.

Los artículos de Ana Moya y Urmila Seshagiri tratan de la primera novela de Orwell, Burmese Days (1934), obra de especial interés para la crítica postcolonial. Así lo parece en el caso de Moya, que establece un análisis de la novela a través de un estudio minucioso de cada personaje, siempre compartiendo con Orwell la perspectiva crítica al sistema colonial ("the corrupting effects of the colonial system", en palabras de la autora, 94). Mediante esta deconstrucción de cada uno de los personajes más importantes, se nos intenta demostrar cómo Burmese Days "is a novel in which issues of race and gender are explored in parallel" (94). La primera obra de Orwell es en realidad una obra de transición, publicada en pleno período de paso desde el sistema colonial inglés al postcolonial. El artículo empieza pues con una explicación histórica situando la obra en su contexto, imprescindible para poder entenderla en su plenitud. El interés de Ana Moya se centra sin embargo en lo que será su postulado más interesante: el lenguaje como medio de dominación, técnica que Orwell conocía bien, como demostró en Animal Farm (1945) o Nineteen Eighty-Four (1949), y con la que fue bastante crítico. Vemos, por ejemplo, cómo el personaje más racista y xenófobo, Ellis, no soporta la idea de unos nativos hablando un "proper English", o cómo se ridiculiza a los euroasiáticos (hijos de padre inglés y madre india), por no hablar el inglés a la perfección. Para una novela colonial, la terrible simbología de la palabra como medio de dominación es sin duda un gran acierto, que Ana Moya descifra y explica con exactitud. La última parte del artículo se centra en un punto de vista feminista para demostrar cómo con Burmese Days Orwell no intentó sino criticar cualquier clase de dominación (Empire-Colony, Men-Women...).

El artículo de Urmila Seshagiri sobre esta misma novela es más explícito en su enfoque feminista. La crítica postcolonial se une aquí al enfoque feminista en un artículo profundamente valiente en el que Seshegari no duda en acusar a Orwell de "ignoring (and 
indeed, naturalizing) the sexual tyranny that oppresses female characters" (106). Comienza, y termina, diferenciando Burmese Days de A Passage to India, para (de)mostrar los defectos de la novela de Orwell; si bien ambas novelas hacen hincapié en cómo la hipocresía del colonialismo contamina las relaciones personales y las decisiones políticas, Burmese Days "fails to consider colonialism's sexual assymetries" (106). Para probar su tesis Seshagiri recurre a los postulados de Gayatri Spivak, a partir de los cuales establece un análisis de los principales personajes femeninos de la obra, Ma Hla May, la prostituta nativa, en yuxtaposición con Elizabeth Lackersteen, inglesa y blanca. Orwell no sale victorioso de este análisis apoyado en la teoría spivakiana y es acusado, como mínimo, de ignorar la situación de la mujer nativa en la India colonial.

La segunda parte del libro, "Orwell, History and Dystopia" se inicia con la trascripción de una conferencia de Zöe Fairbairns sobre su novela Benefits (1979). En principio, se podría pensar que en un libro sobre Orwell un ensayo como éste está fuera de lugar. Sin embargo, Fairbairns demuestra su interés por Orwell y la profunda influencia de éste en su obra, no sin ser bastante crítica con él y aclarando desde el principio su postura distante con muchos de los postulados expuestos en Nineteen Eighty-Four y, sobre todo, en The Road to Wigan Pier (1937). Cuando menos, al finalizar este artículo el lector siente interés por acercarse a la obra de esta autora feminista, admiradora de Doris Lessing y revisora excepcional de la obra de Orwell.

La segunda parte continúa con el artículo de Beatriz Domínguez García "The Retelling of History Through Her Story". Es éste un artículo especial dentro del volumen, pues el nombre de Orwell no aparece ni una sola vez. Se trata de un análisis comparativo entre dos novelas, Behind the Scenes at the Museum (1995) de Kate Atkinson y Stand We at Last (1983) de la ya comentada ZoëFairbairns. A partir de esta comparación entre dos novelas de corte feminista pero con distinta concepción y escritas con una década de diferencia, vemos cómo la reescritura de la historia desde un punto de vista feminista ("herstory" versus "history") demuestra la ausencia de la mujer en la tradición literaria. También señala Domínguez García cómo el activismo feminista (literario o no) "obliges them [women] to transform their personal lives into a political issue or their political ideas into their personal lives" (155).

"Looking Backwards and Fordwards from Nineteen Eighty-Four: Women Writing Men's Worlds", de Elizabeth Russell es otro de los artículos que utilizan a Orwell como excusa para profundizar en otros autores. En este caso, se estudian cuatro novelas desde la óptica feminista y siempre atendiendo a la distinción "utopia-dystopia". Todas ellas están escritas por mujeres, pero narradas por hombres. Las dos primeras novelas, escritas dentro de épocas y culturas diferentes, se acercan al Apocalipsis desde la perspectiva del último hombre (que no mujer) vivo: The Last Man (1826) de Mary Shelley y Memòries d'un Futur Bàrbar (1975) de Montserrat Julió. La aproximación a estas pesadillas futuristas se hace siempre desde términos puramente postmodernistas. Así, Russell indaga en la subjetividad de vocablos tales como "historiografía" o "biografía". Parece estar muy interesada la autora en lo subjetivo de la capacidad del individuo de plasmar su realidad, en nuestra imposibilidad de controlar el lenguaje: "Writing history, writing about self, always requires a relationship of complicity with language (...) and language always falls short of meaning" (158). Parece que si plasmar con la palabra los hechos del pasado es algo fuera de nuestro alcance, imaginar los del futuro es más difícil aún. La objetividad, para el historiador o el escritor, es un reto quimérico que no es necesario en la ciencia ficción. Y éste es un punto de partida muy interesante para la crítica feminista, que puede encontrar en este género lo que falta en la novela realista.

Odisea, $n^{\circ}$ 2, 2002 
También insiste Russell en definir y diferenciar los términos "utopia" vs. "dystopia". "A utopia is a vision of an ideal society (...) Utopia is always elsewhere, but never here. Although it indirectly constitutes a critique of the society in which it is constructed, utopia is invariably so different from reality as to be unrealisable. A dystopia, on the contrary, is often easily imaginable and recognisable and constitutes a warning..." (160). Esta perfecta definición es aplicable a la obra de Orwell (no sólo a Nineteen Eighty-Four), quien muy probablemente hubiese coincidido con Russell.

Las otras dos novelas consideradas en este artículo son Man's World (1926) de Charlotte Haldane y Swastika Night (1937) de Katherine Burdekin, ambas escritas en el periodo de entre guerras. No es casualidad que la novela de Burdekin fuese publicada por Victor Gollancz, editor también de Orwell, quien vería en esta fábula anti-fascista un libro ideal para su Left Book Club. Russell recurre a Barthes para aproximarse al estudio del mito en estas novelas como sustitución de la realidad histórica, y a Kristeva para indagar en el lenguaje como elemento extraño al individuo, recurso frecuente en la literatura futurista, incluyendo al propio Orwell. Russell se aleja aquí de su visión postmodernista para poder explorar la búsqueda de la verdad que llevan los personajes de estas obras, frente a la mentira que siempre encuentran.

En "'History is not a Thing of the Past': The Theory and Practice of Historical Discourse in Alasdair Gray's A History Maker" Sonia Villegas sigue analizando el legado de Orwell en la narrativa más reciente. Intenta demostrar cómo los puntos en común entre el autor escocés y Orwell son más que patentes. Para ello comienza indagando en el concepto de historia y su importancia para ambos autores. Villegas baraja con precisión una serie de conceptos (posthistory, the end of history, historiography) para definir la historia como uno de los vocablos cardinales de la cultura postmodernista, convirtiendo a Orwell en un escritor adelantado a su tiempo. Ambos autores parecen haber compartido un especial interés por el uso de la historia como elemento de dominación por parte de las clases en el poder. También comparten una visión no linear del concepto de "tiempo" y un profundo interés por la imposibilidad de alcanzar un conocimiento de la historia completo y objetivo ("the 'fictiveness' of history" que tanto interesó a Orwell). Un gran acierto de Villegas es su reflexión sobre la relación historia-literatura, preocupación muy cercana sin duda al pensamiento orwelliano: "From a conventional viewpoint, history and the novel have depended on one another. Like historical time, narrative time has been considered a progression from a beginning to an end, usually heading towards death, which is transcended through the act of reading" (183).

Otras analogías entre estos dos autores, como descubre Villegas, son su proximidad a los círculos de izquierdas o su pasión por la clase media obrera. El anteriormente debatido nacionalismo de Orwell también es estudiado por Villegas, para compararlo con el diferente nacionalismo escocés de Alasdair Gray. Muy sensatamente, el artículo no termina sin aclarar que, si bien la influencia de Orwell es vital en la obra de Gray, también son muchos los puntos que los distancian, destacando cómo "gender awareness is absent (...) from Orwell's work, whose attention is focused on male ethics and values almost exclusively" (196).

Los dos últimos artículos presentan una gran similitud enfocando el mismo tema (el legado de Nineteen Eighty-Four) desde dos perspectivas hermanadas, enriqueciéndose mutuamente. El capítulo de Juan Francisco Elices "The Satiric and Dystopic Legacy of George Orwell in Robert Harris's Fatherland" nace de la idea con la que él mismo cierra su exposición: la obra de Orwell se ve mejorada si examinamos la herencia de ésta en la literatura contemporánea. Esto lo demuestra a partir de la novela de Robert Harris Fatherland (1992), escrita cuarenta y tres años después de Nineteen Eighty-Four. Comienza analizando los 
posibles motivos que llevaron a Orwell a escribir su última obra y los asemeja con los intereses de Harris. La Europa de Hitler, del miedo y el desempleo que incitó a Orwell a escribir no se distancia tanto de las preocupaciones de Harris. La semejanzas entre las dos novelas son muchas, pero Elices se centra en la crítica al abuso de poder, destacando la distorsión de la historia, la manipulación del lenguaje o los mecanismos de represión, muy presentes en ambas. Aunque al iniciar su artículo Elices promete ajustarse a esos puntos, lo cierto es que continúa tratando muchos más, lo que es de agradecer, pues señala con gran acierto otros aspectos que la literatura de hoy ha heredado de Orwell como la "animalización" del individuo, que la novela de Harris no pasa por alto. Tampoco deja escapar Elices el discutido tema de la represión sexual en la obra de Orwell, para concluir, una vez más, que el autor inglés olvidó a la mujer, lo que no ocurre en la novela de Harris, escrita en los noventa.

El artículo que pone punto y final a este libro es también un perfecto sintetizador de las principales conjeturas con que nos hemos encontrado. Ben Clarke, con un preciso manejo de una amplia bibliografía, rastrea las influencias posibles en la literatura utópica de Orwell (We [1929] de Zamyatin o The Managerial Revolution [1941] de J. Burnham) para concluir que Orwell actualizó el género adaptándolo a una época en la que los estragos del nazismo pesaban sobre cualquier fantasía futurista. No sólo se limita a estudiar los antecedentes de Nineteen Eighty-Four sino que indaga en el legado de esta obra en la ficción posterior. Autores como Ben Elton (The Other End, Gridlock), Julian Barnes (England, England) o el ya estudiado y muy orwelliano Robert Harris y su Fatherland, son para Clarke perfectos ejemplos del renacimiento de la literatura de utopía tras el impacto de Nineteen Eighty-Four.

Este último artículo es además uno de los más ricos en ideas. Clarke también parece estar interesado en la vieja polémica de la interpretación antisoviética de Nineteen EightyFour. Orwell dejó claro que ser reacio al régimen de Stalin no sólo no era incompatible con ser socialista, sino que era imperativo para el buen izquierdista. Clarke demuestra con acierto la forma en que la historia da la razón al Orwell más profético y pone el dedo en la llaga al denunciar cómo la interpretación exclusivista de la obra como una crítica sólo aplicable al régimen soviético descuidó cualquier otra alegoría posible, como por ejemplo la de ser crítica también con el capitalismo o imperialismo feroz.

En resumen, el compendio de artículos del que es editor Alberto Lázaro nos acerca desde muchos puntos de vista a la obra de uno de los escritores sobre los que aún más queda por escribir. El lector que busque conocer la huella que Orwell ha dejado en la literatura de hoy encontrará en este volumen un gran aliado. Quizá en pos de este objetivo se ve mermado el estudio del peso de su obra en sus contemporáneos, aspecto que no descuida Jeffrey Meyers en George Orwell: The Critical Heritage. También se puede echar en falta el olvido en este estudio de una de las que curiosamente han venido siendo las obras más estudiadas de Orwell, como la célebre Animal Farm, a la que apenas se hacen referencias. Lo que no se descuida son los nuevos enfoques de la crítica literaria actual, incluidas las nuevas revisiones feministas. En definitiva, nos encontramos con este libro ante un repertorio de artículos que, pese a tratar a un mismo autor desde perspectivas distintas, se esfuerza por presentar una coherencia entre sí, y que sin duda enriquece la creciente bibliografía sobre uno de los indiscutibles genios creadores de su siglo. 


\section{BIBLIOGRAFÍA}

Meyers, J., ed. 1975. George Orwell: The Critical Heritage. London: Routledge \& Kegan Paul.

Orwell, G. 2000. An Age Like This. 1920-1940. Essays, Journalism and Letters, Volume

I. Eds. I. Angus \& S. Orwell. New York: Brace \&World Inc. 